\title{
Miniaturized Ionic Polarization Diodes for Neurotransmitter Release at Synaptic Speeds
}

Theresia Arbring Sjöström, Amanda Jonsson, Erik Gabrielsson, Magnus Berggren, Daniel Simon and Klas Tybrandt

The self-archived postprint version of this journal article is available at Linköping University Institutional Repository (DiVA):

http://urn.kb.se/resolve?urn=urn:nbn:se:liu:diva-162499

N.B.: When citing this work, cite the original publication.

Arbring Sjöström, T., Jonsson, A., Gabrielsson, E., Berggren, M., Simon, D., Tybrandt, K., (2019), Miniaturized Ionic Polarization Diodes for Neurotransmitter Release at Synaptic Speeds, ADVANCED MATERIALS TECHNOLOGIES, , 1900750. https://doi.org/10.1002/admt.201900750

Original publication available at:

https://doi.org/10.1002/admt.201900750

Copyright: Wiley (12 months)

http://eu.wiley.com/WileyCDA/ 


\section{WILEY-VCH}

DOI: $10.1002 /(($ please add manuscript number $))$

Article type: Communication

\section{Miniaturized Ionic Polarization Diodes for}

\section{Neurotransmitter Release at Synaptic Speeds}

Theresia Arbring Sjöström, Amanda Jonsson, Erik O. Gabrielsson, Magnus Berggren, Daniel T. Simon* and Klas Tybrandt*

T. Arbring Sjöström, Dr. A. Jonsson, Dr. E. O. Gabrielsson, Prof. M. Berggren, Assoc. Prof.

D. T. Simon, Assoc. Prof. K. Tybrandt

Laboratory of Organic Electronics, Department of Science and Technology, Linköping

University, 60174 Norrköping, Sweden

E-mail: klas.tybrandt@liu.se, daniel.simon@liu.se

Keywords: iontronics, bioelectronics, ion diodes, controlled release, neurotransmitters

\section{Abstract}

Current neural interfaces rely on electrical stimulation pulses to affect neural tissue. The development of a chemical delivery technology, which can stimulate neural tissue with the body's own set of signaling molecules, would provide a new level of sophistication in neural interfaces. Such technology should ideally provide highly local chemical delivery points that operates at synaptic speed, something that is yet to be accomplished. Here we report on the development of a miniaturized ionic polarization diode that exhibits many of the desirable 


\section{WILEY-VCH}

properties for a chemical neural interface technology. The ionic diode shows proper diode rectification and the current switches from off to on in $50 \mu$ s at physiologically relevant electrolyte concentrations. A device model is developed to explain the characteristics of the ionic diode in more detail. In combination with experimental data, the model predicts that the ionic polarization diode has a delivery delay of $5 \mathrm{~ms}$ to reach physiologically relevant neurotransmitter concentrations at subcellular spatial resolution. The model further predicts that delays of $<1 \mathrm{~ms}$ can be reached by further miniaturization of the diode geometry. Altogether, the results show that ionic polarization diodes are a promising building block for the next generation of chemical neural interfaces.

Technology for highly local chemical modulation of neurons enables a new generation of therapies for neurological disorders such as epilepsy and Parkinson's disease. In contrast to electrical stimulation, chemical stimulation can directly access the specific chemical pathways of the neurons, thereby enabling more refined neuromodulation including up- or downregulation. Chemical delivery can be achieved by the manipulation of liquids, ${ }^{[1-3]}$ although such approaches often struggle with miniaturization, addressability, and integration into compact freestanding units. An alternative technology that circumvents several of these issues is iontronics, ${ }^{[4]}$ in which ionic fluxes of chemicals are directly controlled. Favorable characteristics of iontronic devices are the absence of moving mechanical parts, miniaturization through microfabrication, direct electronic control, and essentially no induced convection. The organic electronic ion pump (OEIP) ${ }^{[5]}$ is an iontronic device developed for the delivery of ions and charged biomolecules. In its most basic form, the OEIP includes two reservoirs with driving electrodes and a channel comprising selective ion exchange material. 


\section{WILEY-VCH}

By applying a voltage between the two electrodes, the desired ionic species (ions or small charged biomolecules) can be selectively transported from the reservoir to the target, thereby providing electronically controlled chemical delivery. Freestanding ${ }^{[6-8]}$ and implantable OEIPs have been demonstrated and used in animal models for the treatment of pain ${ }^{[9]}$ and epilepsy ${ }^{[10]}$. When interfacing the nervous system, high spatiotemporal control of the delivery enables reduced dosage levels, thereby reducing or even eliminating unintended side effects elsewhere in the body. When the aim is to replace or bypass neural signals, the time from decision to actual delivery needs to match the speed of synaptic release and transport, which is on the order of milliseconds. ${ }^{[11]}$ To achieve such delivery speed, two challenges must be met simultaneously. First, the neurotransmitters must be stored close to the delivery target. Since these types of delivery devices preferably are fabricated in thin films, short distances are readily available in the vertical direction. A vertical release path implies that a steep concentration gradient is necessary, which results in an intolerably high passive leakage unless active measures are taken to avoid it. This second challenge requires the use of nonlinear ionic components, e.g. nanofluidic $\operatorname{diodes}^{[12,13]}$ and transistors ${ }^{[14]}$, or ionic bipolar $\operatorname{diodes}^{[15,16]}$ and transistors ${ }^{[17]}$. Ionic bipolar diodes have been investigated both experimentally and theoretically for this application with promising results. ${ }^{[18,19]}$ Delivery of the neurotransmitter acetylcholine was demonstrated within $50 \mathrm{~ms}^{[18]}$ while device and operation optimization is predicted to deliver neurotransmitters within 5-10 $\mathrm{ms}^{[19]}$. Here we develop and investigate a new type of ionic diode for fast delivery applications, the ionic polarization diode. The diode comprises an encapsulated cation exchange membrane (CEM) feeding channel with a micropatterned high aspect ratio opening incorporated. The device utilizes the concentration-polarization phenomena observed for ion exchange membranes ${ }^{[20]}$ but localizes the effect within the high aspect ratio outlet to achieve a delivery diode. The 


\section{WILEY-VCH}

developed diode is characterized both in terms of current rectification and pulse response, where the pulsed mode is the expected main operation mode for neurological application. In pulsed mode, the delivery mimics vesicle release where a controlled and minimized dose of neurotransmitters can be delivered. A numerical model is developed and compared to the experimental results. The model indicates that the delivery delay time of the presented device is around $5 \mathrm{~ms}$ and shows how to improve the design for future devices to achieve local delivery times below $1 \mathrm{~ms}$.

The delivery device comprises a source reservoir, a waste reservoir, a feeding CEM channel connecting the source to the waste, and a target reservoir connected to the CEM channel by the polarization diode (Figure 1). The feeding CEM channel is made of poly(styrene sulfonate)-co-maleic acid cross-linked with polyethylene glycol (PSS-co-MA/PEG). ${ }^{[21]}$ Electrodes within the reservoirs are used to control the ion flows through the device. The ion/neurotransmitter to be released is placed in the source reservoir and transported laterally to the waste by the applied voltage $V_{W}$. The delivery is performed vertically through the polarization diode, which comprises the bottom CEM channel and the high aspect ratio hole (Figure 1b). The release of the transported species to the target is controlled by the voltage over the diode, which is influenced by the applied voltage $\left(V_{T}\right)$ to the target electrode in relation to the potential in the feeding channel. When the potential is higher at the target side of the diode, it is in reverse bias (Figure 1c). Due to the cation selectivity of the CEM channel, in reversed bias cations enter the CEM while mobile anions are transported away from the membrane-electrolyte interface within the diode. This concentration polarization depletes the interface of mobile ions, which, with time, creates a linear diffusion gradient throughout the hole. The depletion of mobile ions and the high local electric fields at the CEM-electrolyte 


\section{WILEY-VCH}

interface prevents diffusion of ions out of the diode, thereby effectively suppressing passive leakage. The current level where this depletion occurs is called the limiting current. The magnitude of the limiting current in reverse bias depends on the diffusion of cations from the diode outlet (close to bulk target electrolyte concentration) to the CEM in the bottom. The diffusional flux thus scales with the target electrolyte concentration, the lateral size of the diode, and the height of the diode. A high aspect ratio diode therefore has lower reverse bias current than a low aspect ratio diode of the same lateral dimensions. Altogether, this causes the characteristic limiting current behavior of the diode in reverse bias, with only a weak current dependence on the magnitude of the applied voltage. When the potential is lower at the target side of the diode, the concentration next to the CEM in the diode increases as anion transport is blocked through the CEM (Figure 1d). In this mode the diode has low resistance, and the device resistance is typically dominated by the resistances of the lateral channels. The polarization diodes are implemented as $4 \times 4 \mu \mathrm{m}$ or $6 \times 6 \mu \mathrm{m}$ openings through the $10 \mu \mathrm{m}$ thick insulating SU-8 layer. Both sizes of outlets were characterized but since the smaller outlets performed better (Supplementary Figure S1), they are presented here as the main results. High aspect ratio SU-8 processing is challenging from a patterning point of view, ${ }^{[22]}$ and we experienced geometrical imperfections of the smaller outlet channels (Supplementary Figure S2).

The $4 \times 4 \mu \mathrm{m}$ diode was characterized by disconnecting the waste terminal and scanning $V_{T}$ for three different $\mathrm{KCl}$ concentrations in the target electrolyte and $100 \mathrm{mM} \mathrm{KCl}$ in the source electrolyte (Figure 2a). As the diode is in direct contact with the target electrolyte, varying the target electrolyte concentration can provide insights into the operation principles of the diode. The graph shows the expected behavior, with a steep slope for the forward bias $\left(V_{T}>\right.$ 


\section{WILEY-VCH}

0) and a flat response in reverse bias. The limiting current changes with electrolyte concentration, as the concentration gradient within the diode and thus the current depends on the electrolyte concentration in the bulk of the electrolyte. The rectification ratio at $\pm 4 \mathrm{~V}$ was 21, 17 and 11 for the $10 \mathrm{mM}, 30 \mathrm{mM}$ and $100 \mathrm{mM}$ electrolytes, respectively. Due to the presence of coins within the channel from operation in forward bias, no exact analytical relationship can be derived between diode geometry, electrolyte concentration and reverse bias current. To analyze the characteristics of the polarization diode in more detail, a numerical model based on the Nernst-Planck-Poisson (NPP) equations $(1,2)$ was developed:

$\frac{\partial c_{i}}{\partial t}=\nabla\left(D_{c_{i}}\left(\nabla c_{i}+z_{i} f c_{i} \nabla \mathrm{V}\right)\right)$

$-\frac{\varepsilon \nabla^{2} V}{F}=\sum_{i} z_{i} c_{i}+z_{f i x} c_{f i x}$

where $c_{i}$ is the concentration, $D_{i}$ the diffusivity, $V$ the electrostatic potential, $c_{f i x}$ the concentration of fixed charges, $z_{i}$ the charge number, $F$ Faraday's constant, and $f=F / R T$. The NPP equations have previously been successfully applied to iontronic devices ${ }^{[23-25]}$ and are here solved by the finite element method. The fixed charge density was experimentally estimated to 1.5-1.8 M (see Supplementary Figure S3), with 1.5 M being used in the simulations to not overestimate the device performance. The simulated diode characteristics (Figure 2b) match the measured ones well. The experimental and simulated limiting current follows the same trend with respect to electrolyte concentration, although there is a difference in absolute values, possibly due to the geometrical imperfections of the fabricated outlet (Supplementary Figure S2). The weak forward current concentration dependence can be attributed to coions entering the channel, as the amount of entering coions depends on the local concentration at the bottom of the diode, which in turn depends on the bulk target electrolyte concentration. The simulated concentration profile around the diode in reverse bias is shown in Figure 2c. At the bottom of the diode the concentration approaches zero, while at 


\section{WILEY-VCH}

the outlet the concentration is close to the bulk target electrolyte concentration. In forward bias, the concentration is instead highest at the bottom of the diode (Figure 2d). The concentration profiles along the z-direction are shown in Figure 2e. The steepness of the concentration gradient scales with the bulk electrolyte concentration, resulting in the differences in limiting current. The potential gradient extends further out through the diode for lower concentrations (Figure 2f), although no significant potential drop occurs outside of the hole for $c \geq 30 \mathrm{mM}$.

The measurements and simulations above show that the polarization diode exhibits proper diode characteristics. For drug delivery applications, however, it is the fast pulsing characteristics for the intended neurotransmitter (here acetylcholine, ACh) that are of interest. This mode of operation is designed to mimic synaptic vesical release and to achieve relevant physiological concentrations ${ }^{[26]}$ for single neurons. For pulsed measurements biologically relevant electrolyte concentrations were used; the source electrolyte was $100 \mathrm{mM} \mathrm{AChCl}$ and the waste and target electrolytes were $100 \mathrm{mM} \mathrm{NaCl}$. First, the proper operation voltage was determined by setting $V_{T}=5 \mathrm{~V}$, scanning $V_{W}$, and observing at which point the diode entered reverse bias (Figure 3a). The transition occurred at $V_{W} \approx 10 \mathrm{~V}$, thus for $V_{T} \approx V_{W} / 2$. The on and off voltages for $V_{T}$ were set to 7 and $2 \mathrm{~V}$, respectively, giving a safety margin of $3 \mathrm{~V}$ for the off state. Next, $V_{W}$ was set to $10 \mathrm{~V}$ and on-pulses $\left(V_{T}\right)$ of various length were applied, and the resulting diode currents were measured (Figure $3 \mathrm{~b}$ ). The on-current rise time was $\approx 50 \mu \mathrm{s}$, followed by a fast decay and then a slower rise again. The off-current showed a different behavior, with an initial current plateau followed by a decay. For longer on-current pulses, more ions are injected into the diode, resulting in longer off-current plateaus to extract the injected ions. The simulated pulse response (Figure 3c) shows a similar behavior but with 


\section{WILEY-VCH}

three noticeable differences: $(i)$ there is no initial spike in on-current, (ii) the on-current reaches the plateau much faster, and (iii) the off-current level does not depend strongly on the on-pulse length. We suspected that the initial spike in the measured on-current is due to capacitive charging through the channel insulator (10 $\mu \mathrm{m} \mathrm{SU-8).} \mathrm{To} \mathrm{test} \mathrm{this} \mathrm{hypothesis,} \mathrm{a}$ simple electrostatic model was developed in which the SU-8 was modelled as a dielectric and the channel and target electrolytes modelled as conductors (Figure S4). After assigning the known material parameters, the resulting simulated current matches the measurement very well (Figure 3d), indicating that the initial spike indeed is capacitive in nature. The experimentally observed slow rise in on-current and differences in off-current levels cannot be explained by the model using the current parameter set. However, by assuming a lower fixed charge density in the channel material in close vicinity of the diode, the observed trend can be reproduced (Figure S5). The lower density of fixed charge would make the conductivity of this region highly dependent on the local ion concentration, which would result in the experimentally observed trends of slowly raising on-currents and higher offcurrents for longer on-pulses. At this stage we cannot conclude if the proposed explanation is the cause of the experimentally observed characteristics, we can only show that it is consistent with the observed experimental trends.

Fast rise in current does not mean that the delivered substance actually reaches the outlet of the diode in a short time. To investigate whether this was the case, simulated ACh concentration profiles around the diode are plotted for a $4 \mathrm{~ms}$ pulse staring at $t=0$ (Figure 3e). At $4 \mathrm{~ms}$, the delivered ACh has reached approximately halfway through the hole. When the pulse ends, the concentration at the bottom starts to decrease due to the reverse current, while at the same time ACh diffuses up through the hole. The ACh concentrations within the 


\section{WILEY-VCH}

diode for various times are shown in more detail in Figure S6. Figure $3 \mathrm{f}$ shows how different pulse times effect the ACh concentration at the outlet (i.e. at the position of the yellow dot in Figure $3 \mathrm{e})$. The time $\left(t_{t h}\right)$ it takes to reach a physiologically relevant concentration, here taken as $100 \mu \mathrm{M},{ }^{[26,27]}$ decreases with pulse length down to $\approx 5 \mathrm{~ms}$ for a $6 \mathrm{~ms}$ pulse. The fast release comes at a cost, as the overshoot in concentration also increase with time, which can be problematic for some applications and limits the frequency with which the interfaced system can be stimulated. Another important metric for delivery systems is the ratio between concentrations when the delivery is on or off. Here the simulated delivery on-off ratio was calculated as the $\mathrm{ACh}^{+}$flux out of the diode after a $6 \mathrm{~ms}$ on pulse divided by the flux in the off steady state. The on-off ratio exceeds $10^{10}$, thus in theory the diode has the ability to essentially block all passive diffusional leakage. In practice, however, the on-off ratio might differ significantly from the theoretical value due to device imperfections, continuous on-off switching, and model idealizations.

As a delivery delay of $5 \mathrm{~ms}$ still does not match synaptic speed, we further investigated how scaling of the diode geometry would affect the delivery delay. As only reducing the diode thickness would increase reverse current drastically, we chose to scale the whole diode geometry instead. Diodes with the same aspect ratio but of varying height $(10,5,2.5 \mu \mathrm{m})$ were simulated for a 6 ms pulse (Figure 4a). Reducing the diode dimension decreases the delay in two ways; the ACh needs to travel a shorter distance through the diode, and the current density increases through the smaller diode cross-section, as the on-current remains similar (channel resistance limited). The resulting concentration at the outlets with respect to time is shown in Figure $4 \mathrm{~b}$, and the extracted time to reach the $100 \mu \mathrm{M}$ threshold shown in Figure 4c. The simulations indicate that the delivery delay depends strongly on the diode 


\section{WILEY-VCH}

height, making delivery delay times $<1 \mathrm{~ms}$ achievable for scaled diodes of height $<5 \mu \mathrm{m}$. The linear trend in the log-log plot means that the threshold time scales as a power law with respect to diode height. One should note that the predicted delivery delay times rely on the object to be chemically stimulated (e.g. neuron or other cell) being located directly at the outlet. For fast stimulation it is therefore critical that cells/tissue are located close to the outlet, otherwise diffusional transport through the surrounding electrolyte will constitute the rate limiting factor.

In this work, electrolytes of $100 \mathrm{mM}$ were used to study the performance of the polarization diode for pulsed delivery of $\mathrm{ACh}^{+}$. The salt concentration of cell culture media and physiological solutions is typically within the concentration range of $120-160 \mathrm{mM}$. To predict how the polarization diode performance would change in different media, simulations of pulsed delivery into target electrolytes of $100 \mathrm{mM}$ and $160 \mathrm{mM}$ concentrations were compared (Supplementary Figure S7). Overall, the current levels did not change much but the off current was slightly higher for $160 \mathrm{mM}$. This lowers the $\mathrm{ACh}^{+}$concentration in the channel around the diode, causing the delay time (i.e. time to reach $100 \mu \mathrm{M} \mathrm{ACh}^{+}$ concentration at the outlet) to increase to $6 \mathrm{~ms}$ for $160 \mathrm{mM}$ from $5 \mathrm{~ms}$ for $100 \mathrm{mM}$. Thus, the media the device operates in affects the performance but only to a limited extent, which is expected as the fixed charge density of the CEM is $1500 \mathrm{mM}$, an order of magnitude higher than physiological concentrations.

Neural interfaces, which can match neurons and synapses in terms of spatiotemporal resolution and chemical specificity, remain a futuristic vision. Here we have investigated a novel iontronic component which could be a small step in the direction of realizing such 


\section{WILEY-VCH}

desirable neural interfaces. The developed polarization diode shows promising characteristics, with an estimated delivery delay $<5 \mathrm{~ms}$. Further, by decreasing the dimensions of the diode, delivery delays well below $1 \mathrm{~ms}$ are predicted. Although only a single delivery outlet is demonstrated in this work, devices with a large number of outlets can be designed by including individually addressable electrodes underneath each diode in line with a previously published structure. ${ }^{[18]}$ Altogether, the presented investigation indicates that ionic polarization diodes fulfill many of the necessary requirements for a fast chemical delivery technology for neural interfacing, thus warranting further in vitro and in vivo studies.

\section{Experimental Section}

\section{Device fabrication}

3-glycidoxypropyltrimethoxysilane (GOPS) solution (GOPS $1 \mathrm{ml}$, ethanol $47.5 \mathrm{ml}$, water 2.5 $\mathrm{ml}$, acetic acid $50 \mu \mathrm{l}$ ) was mixed for at least 15 min while 4-inch glass substrates (Specialty Glass Products) were cleaned (acetone, water), baked for $10 \mathrm{~min}$ in $110^{\circ} \mathrm{C}$ and treated with oxygen plasma (Advanced Vacuum Reactive Ion Etch, $\mathrm{O}_{2} 400 \mathrm{sccm}, 250 \mathrm{~W}, 30 \mathrm{~s}$ ).

Immediately after, the glass substrates were immersed in the GOPS solution for $3 \mathrm{~min}$, quickly rinsed in ethanol and baked at $110^{\circ} \mathrm{C}$ for $15 \mathrm{~min}$. Poly(4-styrenesulfonic acid-comaleic acid) (PSS-co-MA, Sigma-Aldrich, $\mathrm{Mw} \sim 20000,25 \mathrm{wt} \%$ in $\mathrm{H}_{2} \mathrm{O}$ ) in $\mathrm{H}^{+}$form (transformed by dialysis), was mixed with polyethylene glycole (PEG, Mw 400) (4 wt\% PSS-co-MA, 1.5 wt\% PEG in water:1-propanol 1:1). 0.2 vol\% GOPS was added to the mixture $15 \mathrm{~min}$ before spin-coating at $1500 \mathrm{rpm}$ and baked at $110^{\circ} \mathrm{C}$ for at least $1 \mathrm{~h}$. Channels were patterned using Shipley S1818 G2 photoresist, MA6-BA6 Süss Mask Aligner, Microposit MF319 developer, reactive ion etching (RIE, O2 100 sccm, CF4 200 sccm, 150 $\mathrm{W}, 95 \mathrm{~s})$ and acetone for photoresist removal. Ion exchange ( $0.1 \mathrm{M} \mathrm{NaCl}(\mathrm{aq}), 3 \mathrm{~min})$ was 


\section{WILEY-VCH}

performed before encapsulation. SU-8 3010 (MicroChem) was spin-coated at $3000 \mathrm{rpm}$, soft baked for 5 min ramping $65-95^{\circ} \mathrm{C}$. After cooling, $0.05 \%$ Electronic Grade Coating (EGC, 3M Novec $^{\mathrm{TM}}$ ) in 7100 Engineering Fluid (EF, 3M Novec ${ }^{\mathrm{TM}}$ ) was spin-coated at $300 \mathrm{rpm}$ and dried for 5 min to protect the mask during vacuum exposure. After $6 \mathrm{~s}$ vacuum exposure and $60 \mathrm{~s}$ post exposure bake at $95{ }^{\circ} \mathrm{C}$, the EGC was removed in EF. Immediately after, the SU-8 was developed in MrDev 600 and hard baked for $15 \mathrm{~min}$ at $110^{\circ} \mathrm{C}$. After encapsulation, a second GOPS layer was deposited following the steps described earlier.

\section{Device characterization}

Devices were soaked in deionized water overnight prior to the measurements with a Keithley 2612 SourceMeter. For diode characterization, a constant $I_{W}$ was applied until resistance stabilized (channel filled with $\mathrm{K}^{+}$). Next, waste was disconnected and $V_{T}$ set to $5 \mathrm{~V}$ for current stabilization. $V_{T}$ was then scanned between 4 to $-4 \mathrm{~V}(250 \mathrm{mV} / \mathrm{s})$. To determine the operation voltages for pulses (Figure 3a), the channel was loaded with $\mathrm{ACh}^{+}$using a constant voltage $\left(V_{W}=10 \mathrm{~V}\right)$ until resistance stabilized. $V_{W}$ was then scanned between 25 to $5 \mathrm{~V}(2 \mathrm{~V} / \mathrm{s})$. For pulse measurements (Figure 3b), $V_{W}=10 \mathrm{~V}$ and a waveform generator (Agilent 33250A) was used to apply $V_{T}(2 \mathrm{~V} / 7 \mathrm{~V}$, pulse lengths $2-6 \mathrm{~ms}, 112 \mathrm{~ms}$ period $) . I_{W}$ was measured over a 10 $\mathrm{M} \Omega$ resistor by an oscilloscope (Agilent Infinium 54830). The measurements are an average of 100 cycles, smoothed (moving average 8 points) and sampled down.

\section{Numerical simulations}

COMSOL Multiphysics 5.3a software was used to solve the partial differential equation system with the finite element method. Tabulated values for diffusivities in electrolyte were used; $D_{C l-}=20.3 \times 10^{-10} \mathrm{~m}^{2} / \mathrm{s}, D_{N a+}=13.3 \times 10^{-10} \mathrm{~m}^{2} / \mathrm{s}, D_{K^{+}}=19.6 \times 10^{-10} \mathrm{~m}^{2} / \mathrm{s}$, and $D_{A C h^{+}}=$ $5.5 \times 10^{-10} \mathrm{~m}^{2} / \mathrm{s}{ }^{[28]}$. Within the channel, effective diffusivity values were used by scaling the 


\section{WILEY-VCH}

electrolyte values by a fixed factor of 0.8 to match the experimental conductivity of the CEM channel. The fixed charge concentration of the CEM channel was set to $1.5 \mathrm{M}$ (in line with experimental measurements (Supplementary Figure S3) and previous work). ${ }^{[19,25]}$ The boundary conditions (BCs) for the target reservoir were bulk electrolyte concentration and electric potential $\left(V_{T}\right)$. Due to the large channel-electrolyte overlap in the source and waste reservoirs, the channel's BCs were set according to Donnan equilibrium concentrations and potentials (thereby assuming fast non-limiting transport at the interface). The major challenge of the highly non-linear 3D model was convergence, which was achieved by meshing the diode carefully (Figure S8). In particular, the CEM-electrolyte interface within the diode had a mesh of $2 \times 10^{-10} \mathrm{~m}$ in the $\mathrm{z}$-direction. Convergence in steady-state solutions was achieved by solving for increasing steps in $c_{f i x}$ concentration, starting from 0 . Transient simulations used steady-state solutions as their initial conditions.

\section{Supporting Information}

Supporting Information is available from the Wiley Online Library or from the author.

\section{Acknowledgements}

The authors thank Dr. Evan Kang for taking the SEM images. This work was financially supported by the Swedish Foundation for Strategic Research, the Knut and Alice Wallenberg foundation, and the Swedish Government Strategic Research Area in Materials Science on Advanced Functional Materials at Linköping University (Faculty Grant SFO-Mat-LiU No. 2009-00971). T.A.S., A.J. and E.O.G. developed the fabrication protocol. T.A.S. fabricated and characterized the devices and analyzed the data. K.T. performed the numerical 


\section{WILEY-VCH}

simulations. K.T., D.T.S. and M.B. guided the project. T.A.S. and K.T. wrote the first draft of the manuscript and all authors contributed to the finalization of the paper.

Received: ((will be filled in by the editorial staff))

Revised: ((will be filled in by the editorial staff))

Published online: ((will be filled in by the editorial staff))

\section{References}

[1] J. M. K. Ng, I. Gitlin, A. D. Stroock, G. M. Whitesides, Electrophoresis 2002, 23, 3461 .

[2] A. Brask, D. Snakenborg, J. P. Kutter, H. Bruus, Lab Chip 2006, 6, 280.

[3] J. Noo Li, D. T. Chiu, C. J. Wargo, W. Hongkai, I. S. Choi, J. R. Anderson, G. M. Whitesides, Biomed. Microdevices 2002, 4, 117.

[4] T. Arbring Sjöström, M. Berggren, E. O. Gabrielsson, P. Janson, D. J. Poxson, M. Seitanidou, D. T. Simon, Advanced Materials Technologies 2018, 3, 1700360.

[5] J. Isaksson, P. Kjäll, D. Nilsson, N. Robinson, M. Berggren, A. Richter-Dahlfors, Nat. Mater. 2007, 6, 673.

[6] D. T. Simon, S. Kurup, K. C. Larsson, R. Hori, K. Tybrandt, M. Goiny, E. H. Jager, M. Berggren, B. Canlon, A. Richter-Dahlfors, Nat. Mater. 2009, 8, 742.

[7] I. Uguz, C. M. Proctor, V. F. Curto, A.-M. Pappa, M. J. Donahue, M. Ferro, R. M. Owens, D. Khodagholy, S. Inal, G. G. Malliaras, Adv. Mater. 2017, 29, 1701217.

[8] D. J. Poxson, E. O. Gabrielsson, A. Bonisoli, U. Linderhed, T. Abrahamsson, I. Matthiesen, K. Tybrandt, M. Berggren, D. T. Simon, ACS Applied Materials \& Interfaces 2019, 11, 14200.

[9] A. Jonsson, Z. Y. Song, D. Nilsson, B. A. Meyerson, D. T. Simon, B. Linderoth, M. Berggren, Sci. Adv. 2015, 1.

[10] C. M. Proctor, A. Slézia, A. Kaszas, A. Ghestem, I. del Agua, A.-M. Pappa, C.

Bernard, A. Williamson, G. G. Malliaras, Sci. Adv. 2018, 4, eaau1291.

[11] B. L. Sabatini, W. G. Regehr, Annu. Rev. Physiol. 1999, 61, 521.

[12] R. Karnik, C. H. Duan, K. Castelino, H. Daiguji, A. Majumdar, Nano Lett. 2007, 7,

547.

[13] L. J. Cheng, L. J. Guo, ACS Nano 2009, 3, 575.

[14] R. Karnik, R. Fan, M. Yue, D. Y. Li, P. D. Yang, A. Majumdar, Nano Lett. 2005, 5, 943.

[15] E. O. Gabrielsson, K. Tybrandt, M. Berggren, Lab Chip 2012, 12, 2507.

[16] H.-R. Lee, J. Woo, S. H. Han, S.-M. Lim, S. Lim, Y.-W. Kang, W. J. Song, J.-M.

Park, T. D. Chung, Y.-C. Joo, J.-Y. Sun, Adv. Funct. Mater. 2019, 29, 1806909.

[17] K. Tybrandt, K. C. Larsson, A. Richter-Dahlfors, M. Berggren, Proc. Natl. Acad. Sci. U. S. A. 2010, 107, 9929. 


\section{WILEY-VCH}

[18] A. Jonsson, T. A. Sjostrom, K. Tybrandt, M. Berggren, D. T. Simon, Sci. Adv. 2016, 2.

[19] K. Tybrandt, Soft Matter 2017, 13, 8171.

[20] V. M. Aguilella, S. Mafe, J. A. Manzanares, J. Pellicer, J. Membr. Sci. 1991, 61, 177.

[21] T. A. Sjostrom, A. Jonsson, E. Gabrielsson, L. Kergoat, K. Tybrandt, M. Berggren, D. T. Simon, ACS Applied Materials \& Interfaces 2017, 9, 30247.

[22] A. d. Campo, C. Greiner, J. Micromech. Microeng. 2007, 17, R81.

[23] N. Boon, M. O. de la Cruz, Soft Matter 2015, 11, 4793.

[24] V. M. Volgin, A. D. Davydov, J. Membr. Sci. 2005, 259, 110.

[25] A. V. Volkov, K. Tybrandt, M. Berggren, I. V. Zozoulenko, Langmuir 2014, 30, 6999.

[26] A. Scimemi, M. Beato, Mol. Neurobiol. 2009, 40, 289.

[27] K. Tybrandt, K. C. Larsson, S. Kurup, D. T. Simon, P. Kjäll, J. Isaksson, M. Sandberg, E. W. H. Jager, A. Richter-Dahlfors, M. Berggren, Adv. Mater. 2009, $21,4442$.

[28] E. M. Sega, C. F. Tormena, P. R. de Oliveira, R. Rittner, L. W. Tinoco, J. D. FigueroaVillar, N. F. Hoehr, J. Mol. Struct. 2006, 797, 44. 


\section{WILEY-VCH}
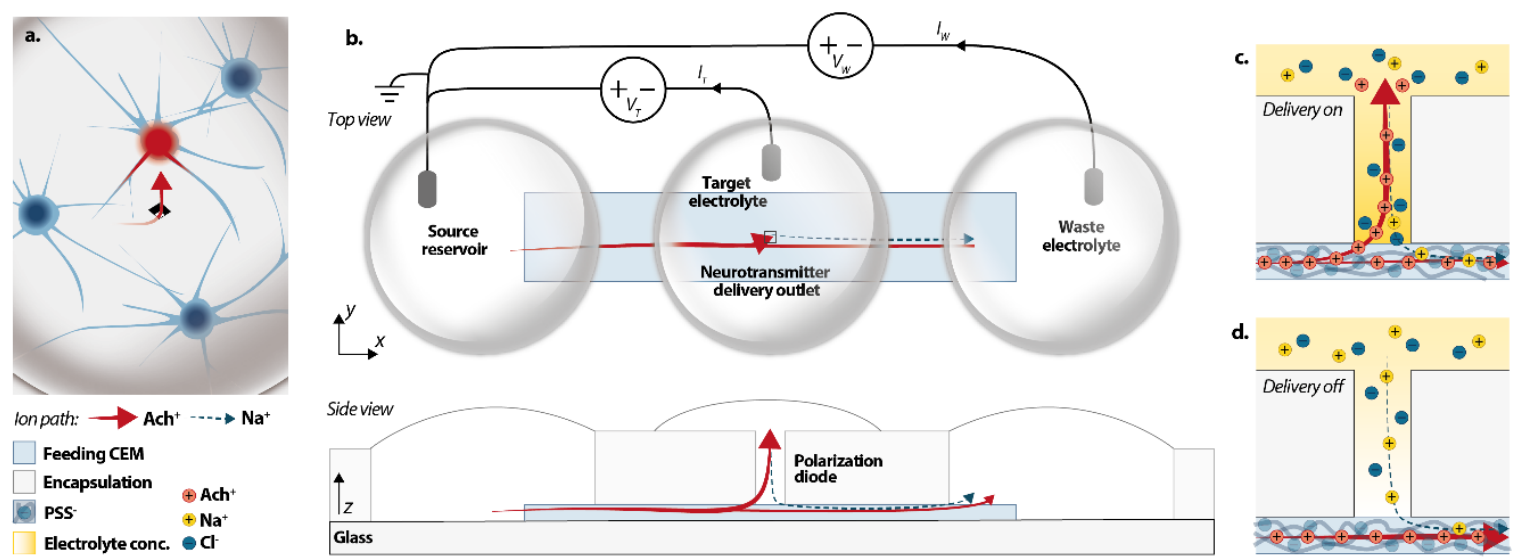

Figure 1. Device schematics. a) The developed delivery system should be able to locally deliver physiologically relevant neurotransmitter concentrations at synaptic speed to neurons (or other cells/tissue) located directly above the delivery outlet. b) The source, waste and target reservoirs are connected by a CEM channel ( $9.5 \mathrm{~mm}$ long, $\approx 200 \mathrm{~nm}$ thick). The ion/neurotransmitter is continuously transported from the source to the waste to fill the channel. The delivery is controlled by the polarization diode ( $4 \mathrm{x} 4 \mu \mathrm{m}$ opening, $10 \mu \mathrm{m}$ in height) to the target reservoir. c) When the target electrode is addressed such that the diode is in forward bias, a large ion current is delivered through the target outlet (delivery on). d) In reverse bias, a small backwards limiting current keeps the outlet depleted of ions and thereby blocks passive leakage (delivery off). 


\section{WILEY-VCH}

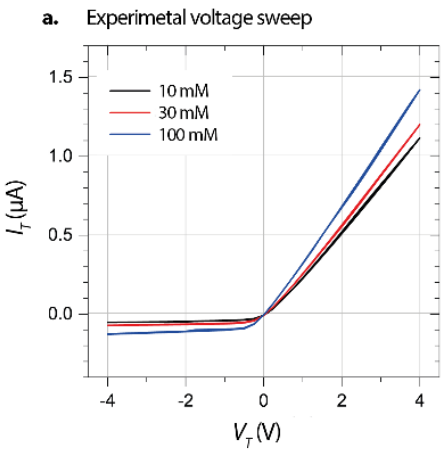

c. Simulated forward bias

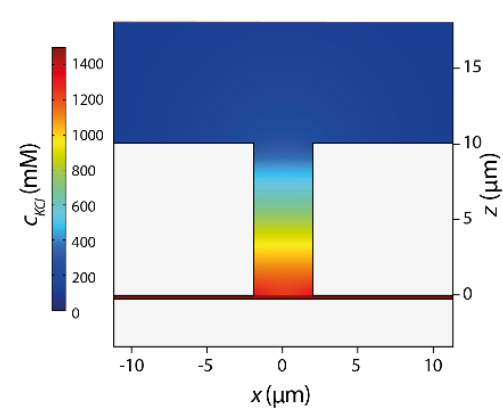

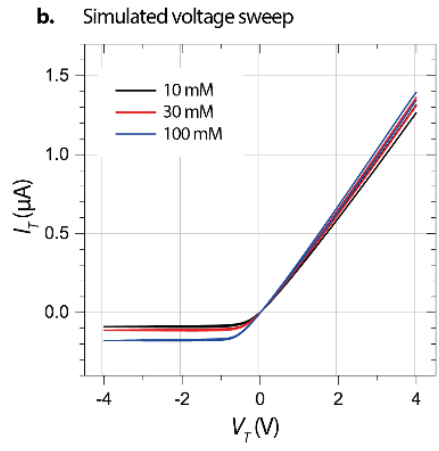

d. Simulated reversed bias

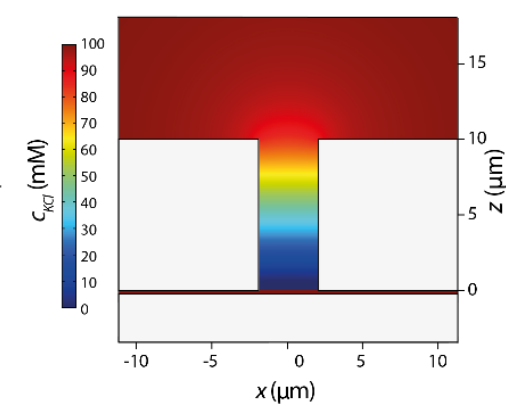

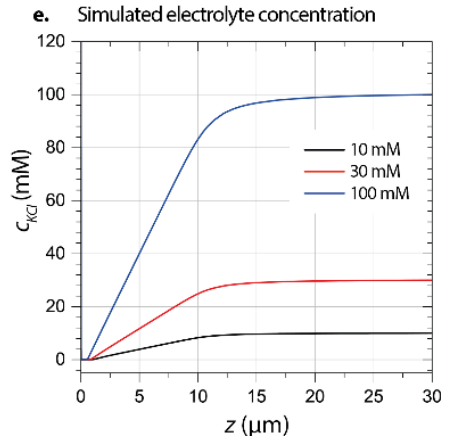

f. Simulated potential drop

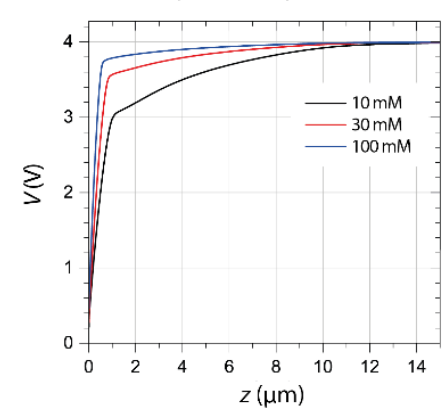

Figure 2. Polarization diode characteristics. a) Voltage sweeps of $V_{T}$ for diodes with different target electrolyte $(\mathrm{KCl})$ concentrations. b) Simulated response of diodes for different target electrolyte $(\mathrm{KCl})$ concentrations. A small hysteresis is visible in the scans. c) Target electrolyte concentration around diode in reverse bias $\left(V_{T}=-4 \mathrm{~V}\right)$. d) Electrolyte concentration around diode in forward bias $\left(V_{T}=4 \mathrm{~V}\right.$, note the different scale). e) $\mathrm{KCl}$ concentration profile along the z-axis of the diode for various target electrolyte concentrations in reverse bias $\left(V_{T}=-4 \mathrm{~V}\right)$. f) Potential distribution along the z-axis of the diode for various $\mathrm{KCl}$ concentrations in reverse bias $\left(V_{T}=-4 \mathrm{~V}\right)$. The source electrolyte was $100 \mathrm{mM} \mathrm{KCl}$ in all measurements. 

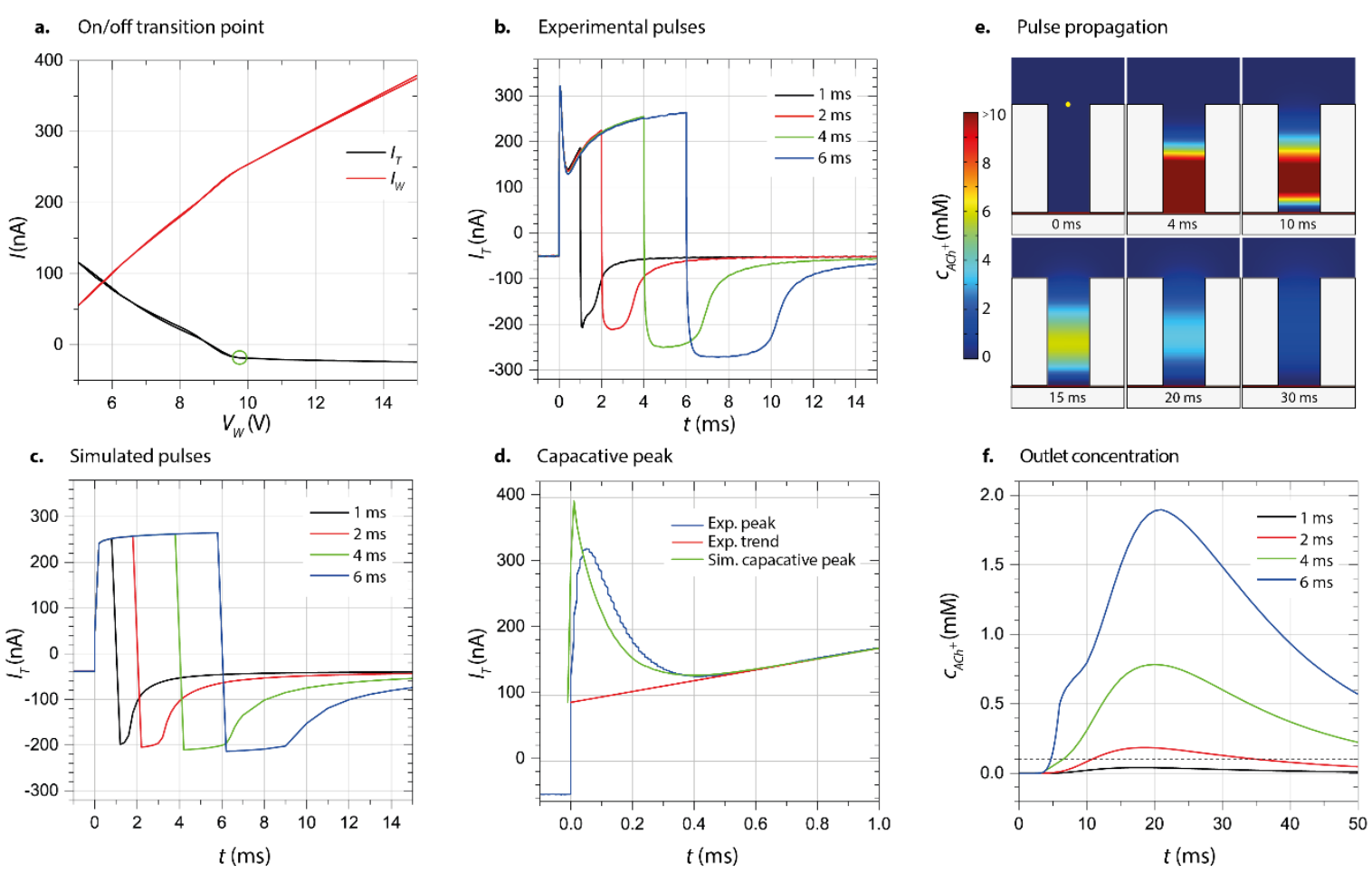

Figure 3. Pulsed delivery. a) To determine around which $V_{T}$ the pulses should be applied, $V_{T}$ was scanned $(2 \mathrm{~V} / \mathrm{s})$ for $V_{W}=10 \mathrm{~V}$. The transition point between forward and reverse bias for ACh delivery is indicated by a green circle. b) The diode was kept in reverse bias by setting $V_{T}=2 \mathrm{~V}$. Delivery pulses of various length were applied by temporarily setting $V_{T}=7 \mathrm{~V}$. The depletion time scales with the pulse length. c) Simulated response of the diode for the same applied voltages as in (b). d) The experimentally measured spike can be reconstructed by adding a simulated capacitive charging current (see SI) to the slower experimental trend in the data. e) Simulated concentration evolution for a $4 \mathrm{~ms}$ long pulse. f) Simulated ACh concertation evolution with time at the outlet (yellow dot in (e)) for pulses of various length. Dashed line indicates a threshold concentration of $100 \mu \mathrm{M}$. All measurements were performed with $100 \mathrm{mM} \mathrm{NaCl}$ in target and waste electrolytes and $100 \mathrm{mM} \mathrm{AChCl}$ in the source electrolyte. 


\section{WILEY-VCH}

a. Pulse propagation in miniaturized diodes

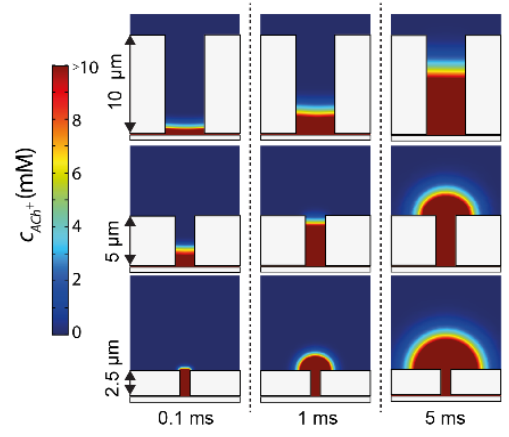

b. Outlet concentration

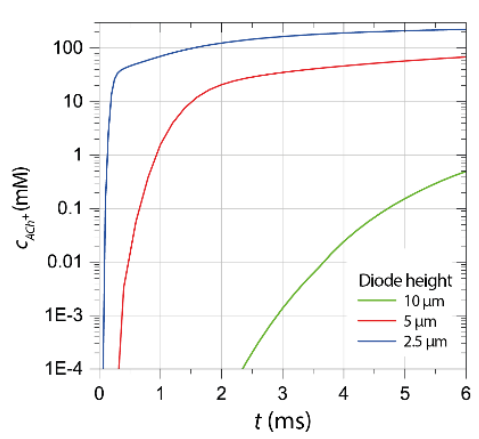

c. Threshold time

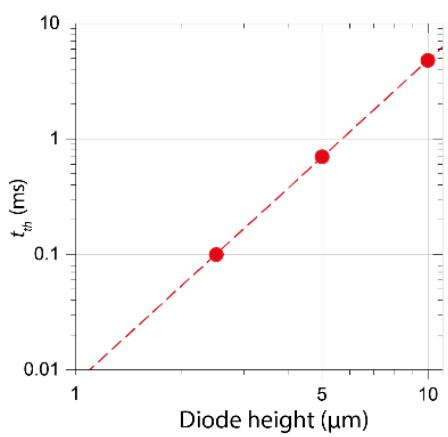

Figure 4. Scaling of the diode geometry. a) Simulated concentration evolution for a $6 \mathrm{~ms}$ pulse for scaled diode geometries of 10, 5 and $2.5 \mu \mathrm{m}$ height, respectively. b) Simulated ACh concentration at the outlet for the different diode geometries as a function of time. c) Threshold time for reaching $100 \mu \mathrm{M}$ concentration at the outlet as a function of diode height. 